\section{No 7050}

IMPLANT THERAPY OUTCOMES, SURGICAL ASPECTS conventional approach in bone grafting procedures

Vucetic V. Milan, Milic S. Marija, Todorovic S. Vladimir, Brkovic M. Bozidar

Department for Oral Surgery, School of Dental Medicine, University of Belgrade

\section{Abstract}

BACKGROUND: In implant dentisty, sufficient bone volume is one of the most important factors to obtain optimal function and esthetics. Traditionaly, intraoral autogenous bone grafts,
considered as a gold stantandard, are predominantly used for alveolar reconstruction. Conversely, harvesting of autogenous bone grafts is associated with additional surgery, costs, morbidity and postoperative discomfort. Finally, to overcome the limitations of intraoral harvesting, the novel minimally invasive piezoelectric technique is introduced in bone grafting procedure. AlM: The aim of this study was to investigate ramus donor site morbidity and postoperative discomfort after intilising piezosurgery compared with conventional techniques prospective study evaluated 19 patients with inadequate bone volume for implant placement in either the maxilla or the mandibule indicated for bone grafting procedures. The split mouth design was achieved using piezoelectric bone surgery technique on one side and conventional technique on contralateral side to obtain ramus bone grafts. Intraoperatively, duration of the osteotomy was recorded in both groups. Postopeartively, time of first pain and first analgesic taken, as well as the first pain intensity were evaluated as a parameters assessed using a visual analogue scale (VAS). For statistical analysis Paired t-test was used and statistical significance was considered as $p<0,05$. RESULTS: The duration of osteotomy was significantly longer during piezosurgery versus conventional tecnique $(p<0.05)$. The time of first pain, as well as time of first analgesic tablet taken were shorter in conventional group in comparison with piezosurgery group, but without statistically significant difference. The first pain intensity (VAS) was not significantly different between
investigated groups $(p>0.05)$. CONCLUSIONS AND CLINICAL IMPLICATIONS: It could be concluded that piezosurgical aproach may be less traumatic for patients postoperatively with respect to the evaluated observations. Regarding that piezoelectric bone surgery could be the recommended prior to implant patient treatment.

\section{Methods and Materials}

A prospective, randomized, spit-mouth, controlled clinical study was performed using a consecutive series of 19 patients, $10(52 \%)$ women and $9(48 \%)$ man, who had mean age of 22,6 years, ranging from 18 to 29 years. The patients were refered to the Department of Oral Surgery (School of Dental Medicine, University of Belgrade, Serbia) with insufficient bone volume for implant placement in either the maxilla and the mandible and scheduled for the bone grafting procedure. Intraoral bone block graft were harvested from the ramus of the mandibule on one side, a bone graft was conventional bone harvesting procedure and on contralateral side using piezosurgical device (Piezotome, Satelec, France)(Fig. 1). Intraoperatively, duration of the osteotomy (expresed in minutes) was recorded in the both groups. Postoperatively, time of first pain and first analgesic tablet taken (expresed in minutes), as well as the first pain intensity were evaluated as a parameters of postoperative discomfort. Time of the first pain and first analgesic tablet taken was considered as a time which is needed from the end of suturing to the first experienced pain and first analgesic tablet taken recorded by patients. The first taken recorded by patients. The firs postoperative pain was assessed using a visual analogue scale (VAS) ranging from 0 (absence of pain) to 10 (most severe pain) on the day of surgery. Statistical analyses were performed using the SPSS version 17 (PASW, USA) Clinical data were expressed as mean \pm SD (Standard Deviation). The paired t-test was used to test any significant differences between the outcomes according to each treatment subgroup and statistical significance was considered as $p<$ 0.05 .

\section{Background and Aim}

Implant reconstruction of patients with partial or complete edentulism has been successful and predictable in cases where sufficient bone volume and adequate bone quality have been achieved $(1,2)$. In that way, autogenous bone grafts, considered as a reference standard for bone reconstruction, are widely used in augmetation procedures because of its osteoconductive, osteoinductive and osteogenetic properties $(3,4)$. Despite these advantages, harvesting of autogenous bone grafts is associated with additional surgery, costs, morbidity and postoperative discomfort (5). Therefore, the use of ultrasonic vibrations to cut bone was first introduced two decades ago to overcome the limitations of conventional instrumentation in oral bone surgery (6). Finally, piezoelectric surgery, as a minimally invasive technique, reduces the risk of damage to the surrounding soft tissues and important structures, such as nerves, vessels, and mucosa, and produce less collateral tissue damage, resulting in better healing response

The aim of the present study was to investigate and compare ramus donor site morbidity and postoperative patients discomfort after utilising piezosurgery compared with conventional instrumentation in bone grafting procedures.
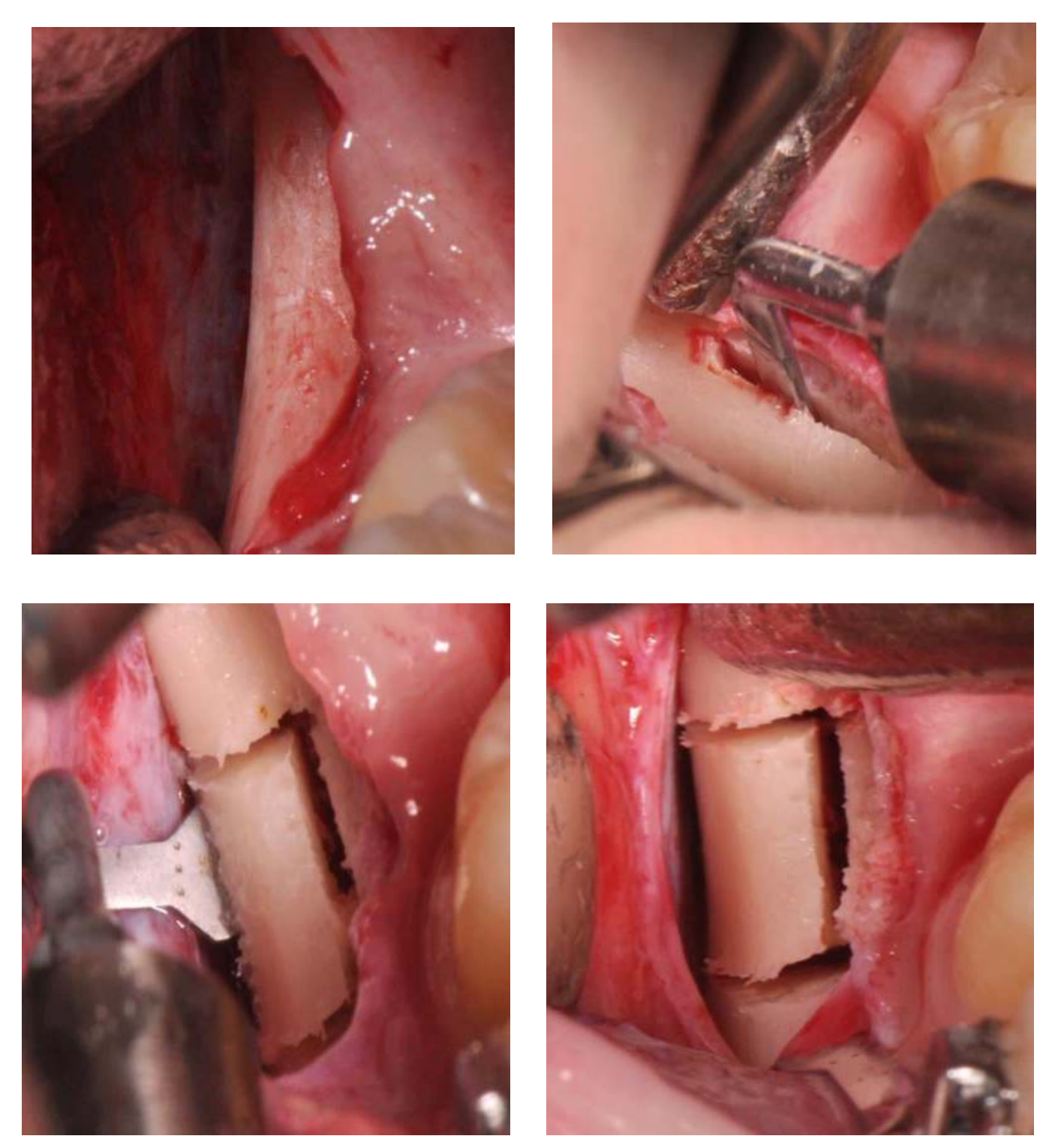

Fig. 1 - Autogenous bone graft obtained from ramus donor site with Fig. 2 - Autogenous bone graft obtained from ramus donor site with
conventional technique (below).
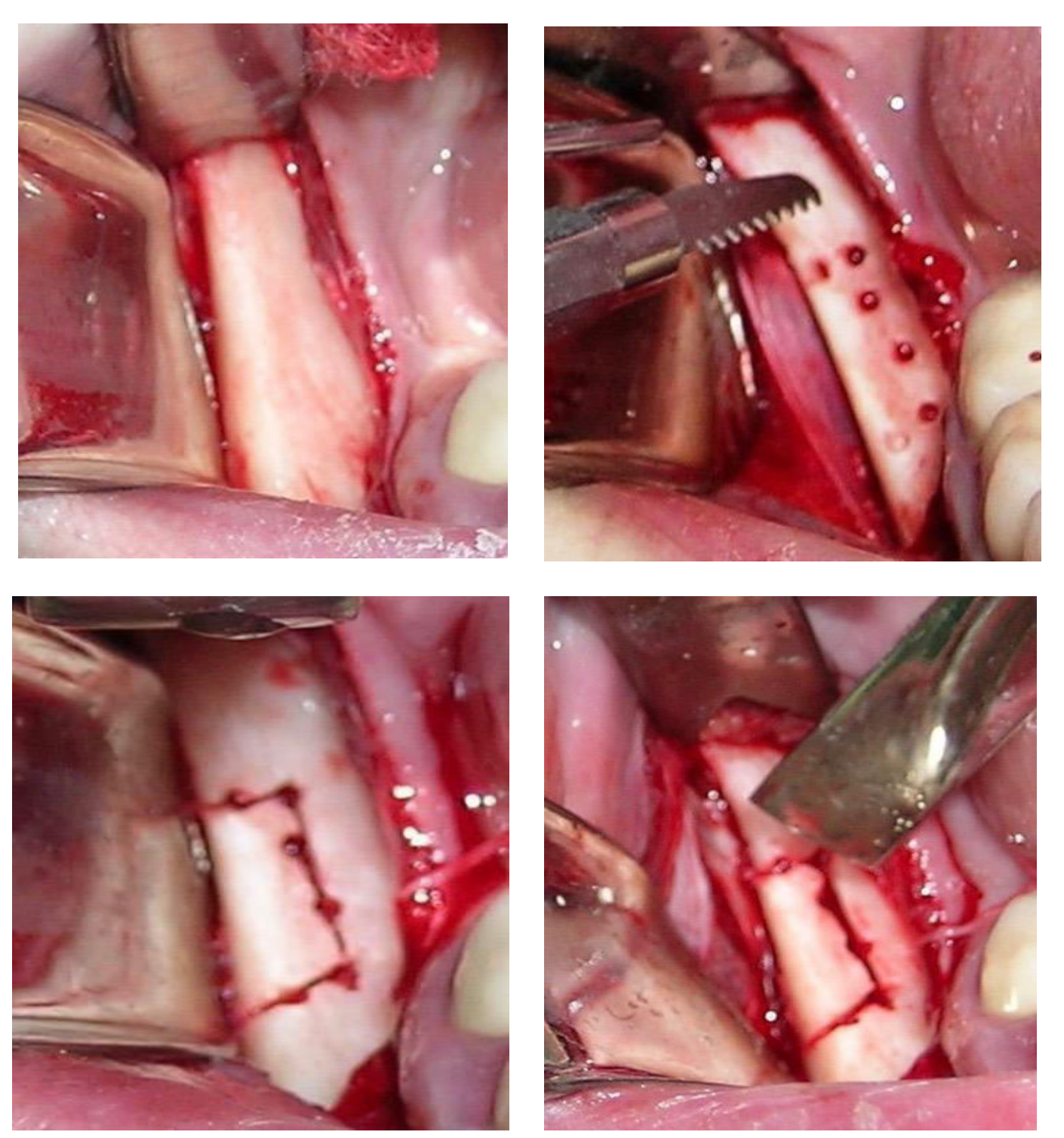

\section{Results}

All donor and recipient sites healed uneventfully.

- There was a significantly longer duration of osteotomy $(p<0.05)$ in the piezosurgery group compared with the conventional group (Table 1).

Table 1. Mean (SD) duration of osteotomy (min) in piezosurgery ( $n=19)$ and
conventional ( $n=19)$ group.

\begin{tabular}{|c|c|}
\hline Groups & $\begin{array}{c}\text { Duration of osteotomy (sec) } \\
\text { (meantSD) }\end{array}$ \\
\hline Piezosurgery group & $3.62 \pm 3.1$ \\
\hline Conventional group & $2.43 \pm 1.0$ \\
\hline P value & 0.045 \\
\hline
\end{tabular}

The time which was necessary to report the first pain, as well as, to take first analgesic tablet was much longer in the group of patient who underwent bone grafting procedures using piezosurgery than that reported after conventional technique, but with no statistical difference (table 2)

- The intensity of the first pain reported by patients was similar in both groups (table 2).

Table 2. Mean (SD) of timing of the first postoperative pain (min), first analgesic
tablet taken (min) and VAS of first pain score $(\mathrm{mm})$ in piezosurgery $(\mathrm{n}=19)$ and

\begin{tabular}{|c|c|c|c|}
\hline Groups & $\begin{array}{l}\text { Time of first } \\
\text { pain }\end{array}$ & $\begin{array}{l}\text { Time of first } \\
\text { analgesic } \\
\text { taken }\end{array}$ & $\begin{array}{l}\text { VAS of the first } \\
\text { pain }\end{array}$ \\
\hline $\begin{array}{l}\text { Piezosurgery } \\
\text { group }\end{array}$ & $161.36 \pm 80.82$ & $234.86 \pm 162.25$ & $43.61 \pm 20.49$ \\
\hline $\begin{array}{l}\text { Conventional } \\
\text { group }\end{array}$ & $146.61 \pm 72.72$ & $184.25 \pm 80.94$ & $37.72 \pm 18.58$ \\
\hline P Value & $P>0.05$ & $P>0.05$ & $P>0.05$ \\
\hline
\end{tabular}

\section{Conclusion}

Based on results of this study, it can be concluded that piezosurgical approach may be less traumatic for patients postoperatively with respect to the evaluated observations, even
though a longer operation time. Regarding that, piezoelectric bone surgery could be recommended as the relevant treatment option in terms donor site morbidity, prior to implant patient rehabilitation

\section{References}

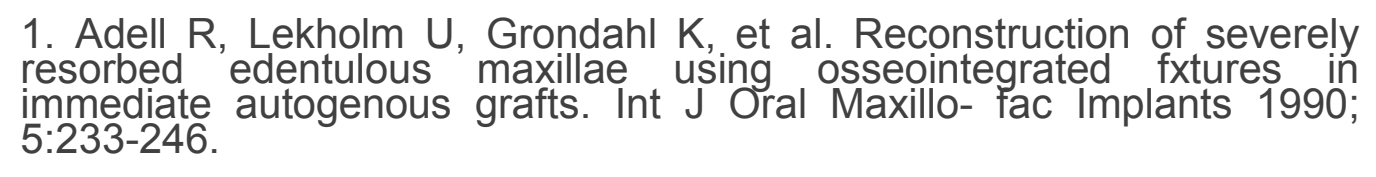
2. Bronemark P-I, Breine U, Lindstrom J et al. Intra-osseous anchorage
of dental prostheses. I. Experimental studies. Scand J Plast Reconstr
Surg 1969; 3:81-100. 3. Jakse N, Seibert FJ, Lorenzoni M, et al: A modified technique of
harvesting tibial cancellous bone and its use for sinus grafting. Clin Oral
lmplants Res $12: 488,2001$ 4. Zaffe D, D'Avenia F: A novel bone scraper for intraoral harvest-ing: A
device for filling small bone defects. Clin Oral Implants Res 18:525, 2007

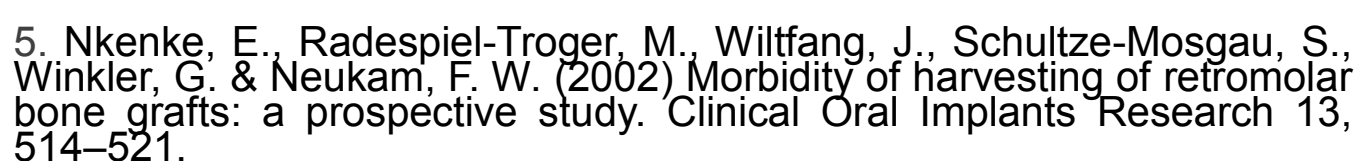
6. Vercellottit T. Technological characteristics and clinical indications of
piezoelectric bone surgery. Minerva Stomatol 2004;53:207-14.

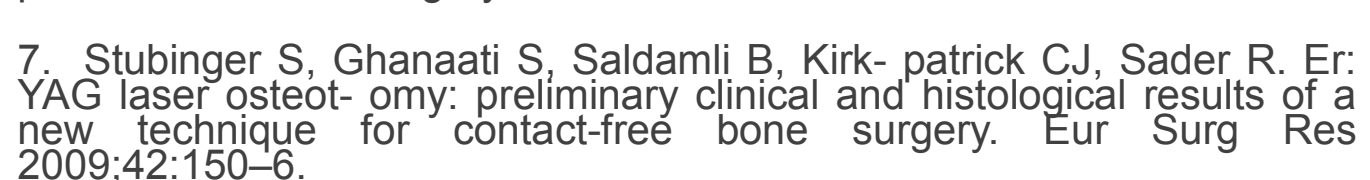

